\title{
AN APPROACH TO THE DELIVERY OF A MICROFOSSIL DATABASE VIA THE INTERNET
}

RODRIGUES*, Cyril G., Dept. of Earth Sciences, University of Windsor, Windsor, ON, N9B 3P4, Canada; KARAMATOS, Stephen, School of Computer Science, University of Windsor, Windsor, ON, N9B 3P4, Canada.

Information in the form of text and graphics is now being disseminated through the World Wide Web (WWW). The information is accessed by personal computers that are connected to the Internet. A WWW site for the delivery of images and distribution data for modern foraminifera and ostracods from the Gulf of St. Lawrence, Canada, is under development. The images were obtained from scanning electron micrographs of the foraminiferal and ostracod species. A small number of images of benthonic foraminifera from cores is also included in the database.

A total of 554 scanning electron micrographs for 183 foraminiferal species and 46 ostracod species comprise the image portion of the database. The micrographs were converted to the Graphics Interchange Format (GIF) for delivery on the WWW. The GIF images are grouped in three modules, Foraminifers in Surface Samples, Foraminifers in Cores and Ostracods in Surface Samples, and they can be viewed in the Browse, Comparison or Search Modes. A menu consisting of a list of genera, that in turn, points to a list of species for each genus, is used to select the image of a species to be viewed under the Browse Mode. The same menu format is used for the Comparison Mode which displays two images of the same species or different species of a genus side-by-side. This mode can be used to compare the morphology of two species of a genus. The Search Mode allows the user to search the database for a species and then to select an image of the species for viewing.

The pages on which the images are displayed under the Browse and Search Modes contain links to the distribution data for the species. The modern distribution data for the species are presented in tables which show occurrences of the species with respect to the water mass subdivisions in the Gulf of St. Lawrence and substrate type. For the benthonic foraminifera, the maximum percent abundance of the species in each water mass subdivision is indicated. The occurrences of the ostracod species in the water mass subdivisions are reported as present or absent because of the low number of ostracod valves in the surface samples. The Uniform Resource Locator (URL) for the site is

http://www.cs.uwindsor.ca/meta-index/mcat/html-docs/woop.html.

The Internet allows researchers and students to access high-quality images and textbased information almost instantaneously and provides a medium for improving the public awareness of paleontological research activities. One advantage of this method of information delivery is the ability to update taxonomic and distribution information, a feature that is not possible with the information provided through the traditional print medium. Also, electronic databases containing images can simplify the task of species identification. 